\title{
Synthetic Approaches to Highly Functional $\beta$-Carboline Building Blocks via Allylic Amidation
}

\author{
Johannes F. Teichert, Martín Fañanás-Mastral, Ben L. Feringa* \\ Stratingh Institute for Chemistry, University of Groningen, Nijenborgh 4, 9747 AG, Groningen, The Netherlands \\ Fax +31(50)3634296; E-mail: b.l.feringa@ rug.nl \\ Received 7 November 2011
}

\begin{abstract}
A new, straightforward synthesis of highly functional $\beta$ carboline building blocks is presented that makes use of allylic amidation methodology. The products obtained carry a terminal double bond as well as an easy-to-deprotect amide, which make them perfectly suitable for further functionalization. The use of the trifluoroacetamide group is exploited in a dual fashion; it acts as a protecting group and functions as the nucleophile for the allylic amidation reaction.
\end{abstract}

Key words: indoles, $\beta$-carbolines, allylic amidation, heterocycles, Stille reaction

$\beta$-Carboline- or tryptoline-derived alkaloids ${ }^{1-3}$ are a large class of compounds that show a wide variety of biological and therapeutic activities (Figure 1). ${ }^{4-6}$ The two antihypertensives, reserpine and ajmalicine, are typical examples of this family of molecules. ${ }^{7-9}$ Many synthetic strategies have been developed to arrive at substituted indoles and tryptamines, ${ }^{10-12}$ with Pictet-Spengler and Mannich type condensation reactions being among the most prominent. ${ }^{13-15}$ However, these methods are limited by the fact that indoles carrying electron-donating groups are required and there are restrictions to the groups that can be introduced via the aldehyde/imine coupling partner.

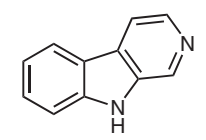

$\beta$-carboline
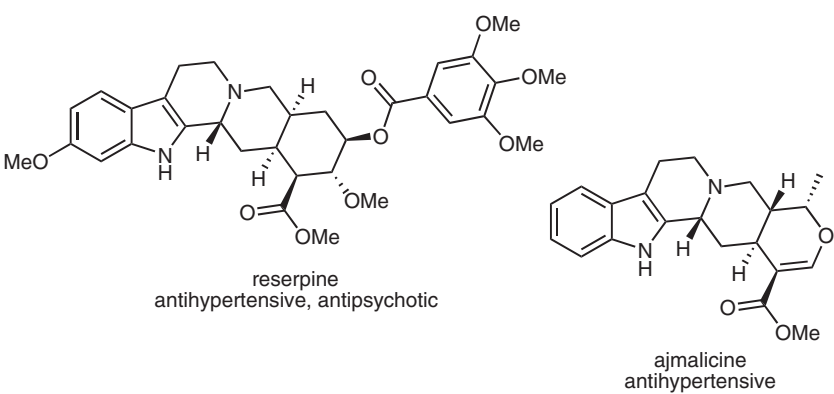

Figure 1 Examples of $\beta$-carboline-derived compounds
We have recently disclosed the first asymmetric intramolecular allylic amidation to give tetrahydroisoquinolines, ${ }^{16}$ based on an iridium-catalyzed allylic substitution reaction ${ }^{17-22}$ with phosphoramidite ligands. ${ }^{23}$ In this protocol, the dual function of the trifluoroacetamide group is crucial, as it serves both as a protecting group and as the actual nucleophile in the key ring-closing transformation. We were interested in expanding this methodology to the synthesis of the important class of $\beta$-carbolines. This reaction should be independent of the electronic demands of the indole moiety and, at the same time, deliver a terminal double bond, which is ideal for further functionalization such as ring annulation (Scheme 1). Together with the amide functionality, which should easily lead to the unprotected amines, our synthetic approach was expected to provide highly functional building blocks for the synthesis of complex molecules featuring the $\beta$-carboline structural unit.

Here, we present a new route to $\beta$-carbolines that exploits the different aspects of reactivity of the trifluoroacetamide group. It was used as a protecting group during a palladium-catalyzed Stille coupling and was also shown to act as a nucleophile in the allylic substitution reaction.

Our synthetic approach started from commercially available tryptamines 1 (Scheme 2). After benzyl protection of the indole nitrogen and trifluoroacetylation of the primary amine, the protected tryptamines $\mathbf{3}$ were obtained in very good yields. Furthermore, trifluoroacetamide 4, without a substituent at the indole nitrogen, was also synthesized.

We then investigated the possible iodination of $\mathbf{3}$ and $\mathbf{4}$ at the 2-position of the indole system. The reported mercurymediated iodination ${ }^{24}$ proved to be feasible on a small scale, however, in our case this proved to be difficult when scaling up the reactions. As an alternative, we explored the use of iodine monochloride as the iodination agent, which we had successfully applied to electron-rich phenylethylamine derivatives. ${ }^{16}$ In the case of tryptamines 3a and 4, this did not prove to be a viable synthetic pathway, because the corresponding chlorides $\mathbf{5}$ were isolated (Scheme 3). The subsequent Stille reaction (see also Scheme 4 below) with stannane $\mathbf{6}$ showed no turnover with chlorides 5 .

The synthesis of the desired iodoindoles 7, which were key intermediates for the envisaged palladium-catalyzed carbon-carbon coupling reaction later on in the synthetic route, was carried out using a lithiation/iodination protocol (Scheme 4). Iodo-substituted indoles 7 were obtained
SYNTHESIS $2012,44,409-416$

Advanced online publication: 22.12.2011

DOI: 10.1055/s-0031-1289656; Art ID: T104711SS

(c) Georg Thieme Verlag Stuttgart · New York 


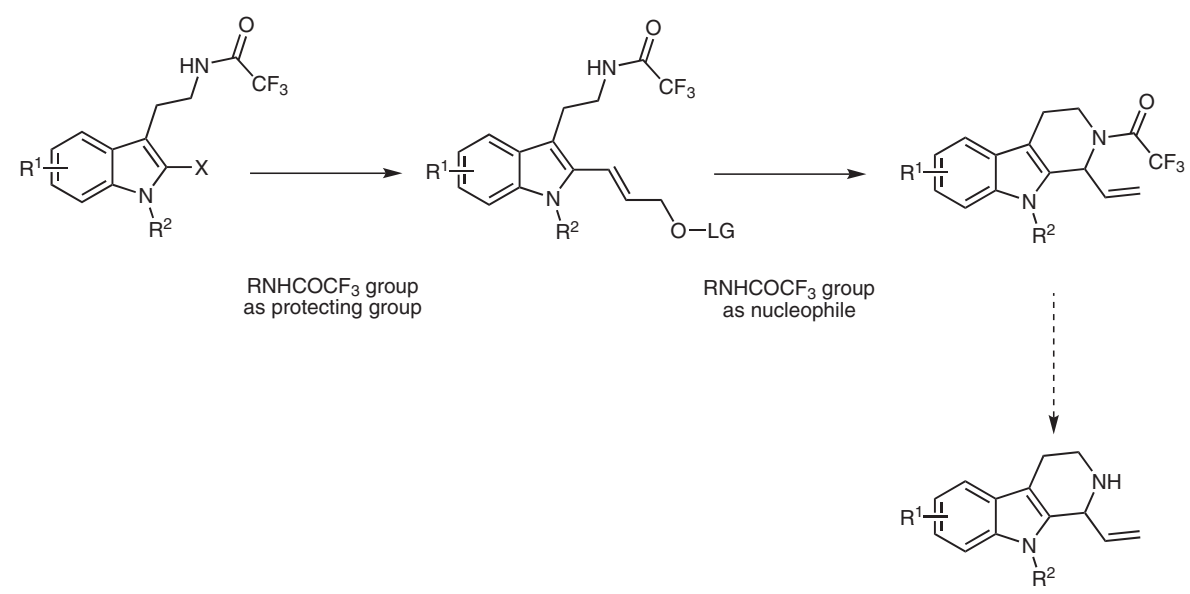

Scheme 1 Synthetic approach

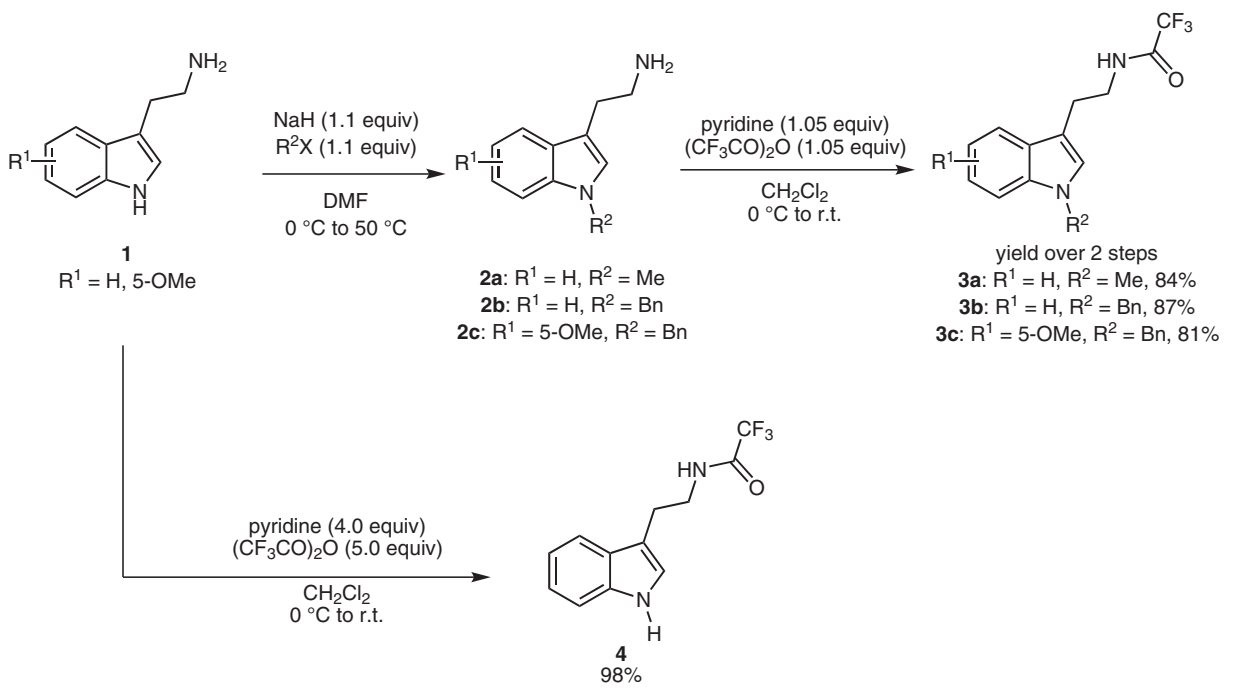

Scheme 2 Synthesis of trifluoroacetamide-protected indoles

in moderate yields. ${ }^{25}$ For the subsequent cross-coupling, the role of the trifluoroacetamide group as a protecting group was exploited. Since palladium-catalyzed coupling only rarely proceeds in the presence of primary amines, this feature could be used to our advantage. In the next step, the allylic alcohol moiety was introduced through Stille coupling with stannane $\mathbf{6}^{26}$ In the case of iodides $\mathbf{7}$, the coupling proceeded smoothly to give the desired allylic alcohols $\mathbf{8}$ (68-71\% yield), which could be transformed into the desired methyl carbonates 9 in a straightforward manner (Scheme 4).

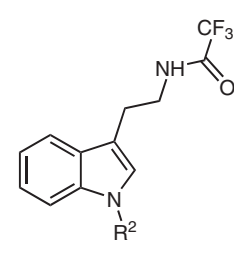

$$
\text { 4: } \mathrm{R}^{2}=\mathrm{H}
$$$$
\text { 3a: } R^{2}=M e
$$

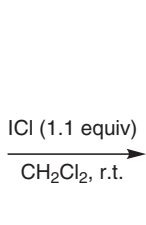

Scheme 3

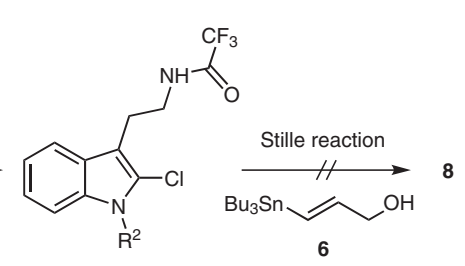

5a: $R^{2}=H, 80 \%$ 5b: $R^{2}=M e, 78 \%$
With the starting materials 9 for the allylic amidation in hand, a method was developed for the selective ring-closing allylic amidation to give $\beta$-carbolines $\mathbf{1 0}$ (Scheme 5). After screening the reaction conditions, it was found that cesium carbonate in dioxane at $100{ }^{\circ} \mathrm{C}$ gave the best outcome for this transformation in terms of yields. When carbonates 9 were heated to $100{ }^{\circ} \mathrm{C}$ in the presence of cesium carbonate, the corresponding tricyclic compounds $\mathbf{1 0}$, bearing a vinyl moiety and an easy-to-deprotect trifluoroacetamide, were obtained in yields of up to $82 \%$. Among the other bases investigated, 1,8-diazabicyclo[5.4.0]undec-7-ene (DBU) and 1,4-diazabicyclo[2.2.2]octane (DABCO) gave low conversion (10 and 50\%, respectively) under the reaction conditions, whereas 1,5,7-triazabicyclo[4.4.0]dec-5-ene (TBD) led to decomposition of the allylic carbonate 9. Other inorganic bases, such as $\mathrm{K}_{3} \mathrm{PO}_{4}$, gave no conversion, whereas the use of sodium hydride as a base led to deprotection of the allylic carbonate to give the corresponding alcohols $\mathbf{8}$. The use of cesium carbonate at lower reaction temperature led to considerably lower conversion of $9\left(10 \%\right.$ conversion at $\left.90{ }^{\circ} \mathrm{C}\right)$, and no reaction took place at $50{ }^{\circ} \mathrm{C}$. It should be noted that the al- 


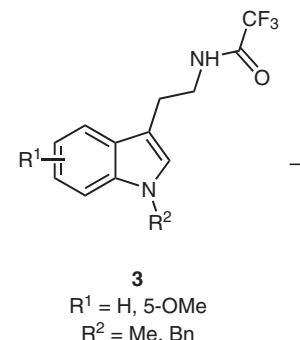

1.) BuLi (2.5 equiv) $\mathrm{Et}_{2} \mathrm{O}, 0^{\circ} \mathrm{C}$ to r.t. 2.) $\mathrm{I}_{2}(1.5$ equiv) $\mathrm{Et}_{2} \mathrm{O}, 0^{\circ} \mathrm{C}$ to r.t. $\mathrm{R}^{2}=\mathrm{Me}, \mathrm{Bn}$
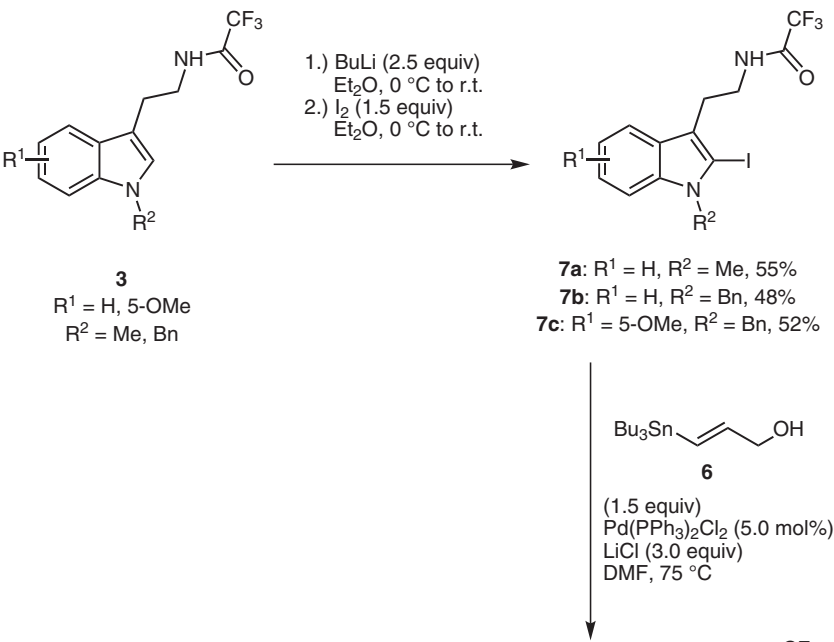

7a: $R^{1}=H, R^{2}=M e, 55 \%$ $7 \mathrm{a}: \mathrm{R}^{1}=\mathrm{H}, \mathrm{R}^{2}=\mathrm{Me}, 55 \%$
7b: $\mathrm{R}^{1}=\mathrm{H}, \mathrm{R}^{2}=\mathrm{Bn}, 48 \%$ 7c: $\mathrm{R}^{1}=5-\mathrm{OMe}, \mathrm{R}^{2}=\mathrm{Bn}, 52 \%$

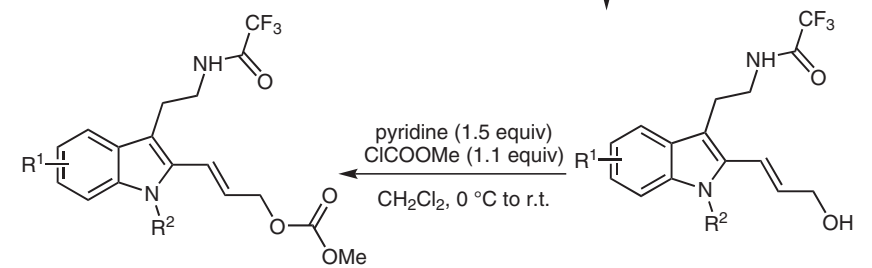

9a: $R^{1}=H, R^{2}=M e, 98 \%$

9b: $R^{1}=H, R^{2}=B n, 98 \%$

9c: $R^{1}=5-\mathrm{OMe}, \mathrm{R}^{2}=\mathrm{Bn}, 91 \%$ 8a: $R^{1}=H, R^{2}=M e, 68 \%$ 8b: $R^{1}=H, R^{2}=B n, 71 \%$ $8 c: R^{1}=5-O M e, R^{2}=B n, 68 \%$

Scheme 4 Synthesis of protected allyl carbonates

lylic amidation could also be carried out by employing microwave heating $(300 \mathrm{~W})$, which led to shortening of the reaction time $(2 \mathrm{~h}$ compared to $16 \mathrm{~h}$ under standard conditions), while retaining similar yields. All attempts to render this transformation asymmetric by the use of iridium ${ }^{16}$ or palladium ${ }^{27}$ catalysis have so far been fruitless.

With this allylic amidation, we have exploited the nucleophilic nature of the trifluoroacetic amide of 9. $\beta$-Carbolines $\mathbf{1 0}$ are highly versatile building blocks for the synthesis of more complex structures, allowing the generation of a variety of molecules featuring a $\beta$-carboline core.

It is interesting to note that products $\mathbf{1 0}$ were isolated as mixtures of isomers/rotamers. Whereas 10a gave rise to two distinct resonances in the ${ }^{19} \mathrm{~F}$ NMR spectrum, which we attribute to the $E / Z$ isomers of the trifluoroacetamide, $10 \mathrm{~b}$ and 10c generated a set of four signals in the ${ }^{19} \mathrm{~F}$ NMR spectra. These additional NMR absorptions were assigned to two rotamers resulting from hindered rotation of the

benzyl protecting group. To probe this hypothesis, we have conducted variable temperature ${ }^{19} \mathrm{~F}$ NMR spectroscopic measurements of 10b (Figure 2). From the data shown in Figure 2 it can be seen that two signals (peaks 1 and 3) coalesce at $80{ }^{\circ} \mathrm{C}$, as expected for rotamers. The fourth signal (Figure 2, peak 4) gradually disappears at higher temperatures, a phenomenon that can be attributed to $E / Z$ isomers since, at higher temperatures, the thermodynamically more stable species (peak 2 at $\delta=-68.2 \mathrm{ppm}$ in Figure 2) should be prevalent in the mixture.

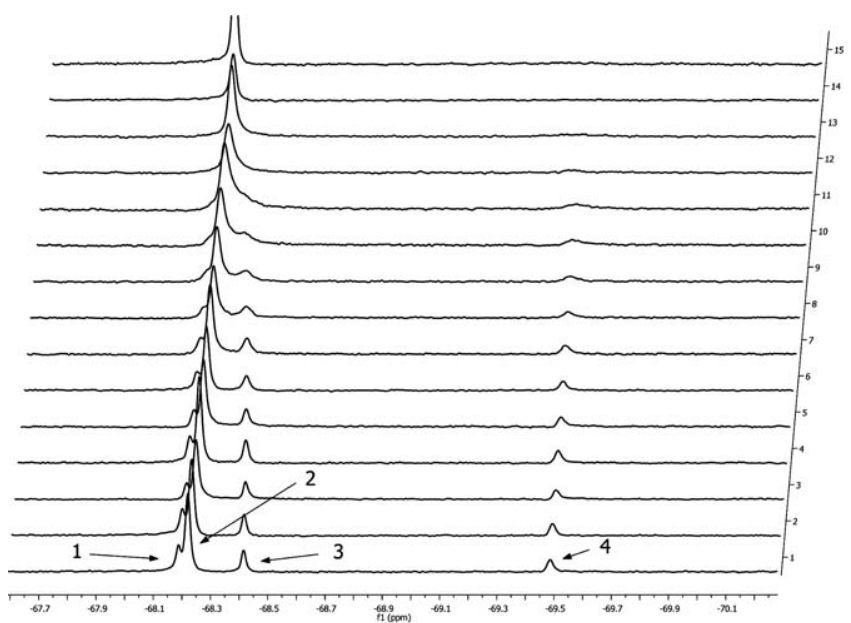

Figure 2 Variable temperature ${ }^{19} \mathrm{~F}$ NMR spectroscopic studies of 10b in DMSO- $d_{6}$; temperature range from $25^{\circ} \mathrm{C}$ (1) to $95{ }^{\circ} \mathrm{C}$ (15) in increments of $5{ }^{\circ} \mathrm{C}$

In summary, we have developed a new, straightforward synthetic pathway towards substituted $\beta$-carbolines, which are important, multifunctional building blocks for further syntheses of complex molecules. In our approach, we exploit the various reactivities of the trifluoroacetic amide moiety as a directing group, a protecting group, as well as a nucleophile in the key allylic amidation step. The products obtained carry a terminal olefin as well as a protected secondary amine, which open up possibilities for a multitude of subsequent transformations, rendering these new building blocks highly versatile.

Merck silica gel type 9385, 230-400 mesh was used for chromatography. Merck silica gel $60,0.25 \mathrm{~mm}$ was used for TLC; components were visualized by UV and cerium/molybdenum staining. Reaction progress and conversion were determined by GC-MS (GC, HP6890; MS HP5973) with an HP1 or HP5 column (Agilent Tech-

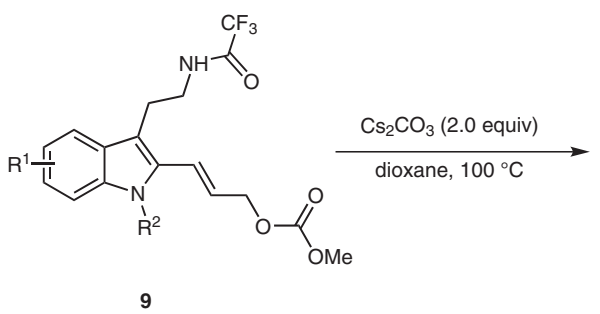

Scheme 5 Synthesis of $\beta$-carboline structures

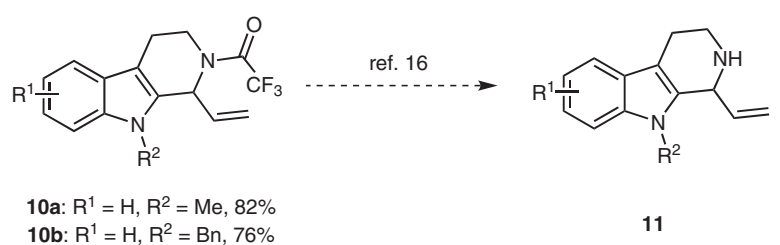

10c: $R^{1}=5-O M e, R^{2}=B n, 82 \%$ 
nologies, Palo Alto, CA, USA). Mass spectra were recorded with an AEI-MS-902 mass spectrometer (EI+) or a LTQ Orbitrap XL (ESI+). ${ }^{1} \mathrm{H},{ }^{19} \mathrm{~F}$, and ${ }^{13} \mathrm{C}$ NMR spectra were recorded with a Varian AMX400 (400 and 100.59 MHz, respectively), a Varian VXR300 (300 and $75 \mathrm{MHz}$, respectively), or a Varian Gemini 200 spectrometer, using $\mathrm{CDCl}_{3}$ as solvent. Chemical shift values $(\delta)$ are reported in ppm with the solvent resonance as internal standard $\left(\mathrm{CHCl}_{3}: \delta=\right.$ $7.26 \mathrm{ppm}$ for ${ }^{1} \mathrm{H}, \delta=77.0 \mathrm{ppm}$ for ${ }^{13} \mathrm{C}$ ). Data are reported as follows: chemical shifts, multiplicity $(\mathrm{s}=$ singlet, $\mathrm{d}=$ doublet, $\mathrm{t}=$ triplet, $\mathrm{q}=$ quartet, $\mathrm{br}=$ broad, $\mathrm{m}=$ multiplet $)$, coupling constants $(\mathrm{Hz})$, and integration. All reactions were carried out under a nitrogen atmosphere, using oven-dried glassware and standard Schlenk techniques. $\mathrm{CH}_{2} \mathrm{Cl}_{2}$ was dried and distilled over $\mathrm{CaH}_{2}$; THF and $\mathrm{Et}_{2} \mathrm{O}$ were dried and distilled over $\mathrm{Na} /$ benzophenone. Toluene was dried and distilled over Na. Stannane $\mathbf{6}$ was prepared according to a literature procedure. ${ }^{26}$

\section{N-Benzylation or N-Methylation of Tryptamines 1 To Give 2; General Procedure}

The appropriate tryptamine 1 (1.00 equiv) was dissolved in DMF $(10 \mathrm{~mL} / \mathrm{mmol})$ at $50{ }^{\circ} \mathrm{C}$ and added to a stirred solution of $\mathrm{NaH}(1.10$ equiv) in DMF $(10 \mathrm{~mL} / \mathrm{mmol})$ and the mixture was stirred for 30 min. $\mathrm{BnBr}$ or $\mathrm{MeI}$ (1.10 equiv) was added dropwise. After stirring at $50{ }^{\circ} \mathrm{C}$ for $1 \mathrm{~h}$, the reaction was quenched with $\mathrm{H}_{2} \mathrm{O}(10 \mathrm{~mL} /$ $\mathrm{mmol})$ and the mixture was extracted with EtOAc $(3 \times 10 \mathrm{~mL} /$ mmol). After drying over $\mathrm{MgSO}_{4}$ and removal of all volatiles, $\mathrm{N}$ substituted tryptamines $\mathbf{2}$ were obtained as yellow/orange solids, which were used without further purification.

\section{Trifluoroacetylation of 2 To Give 3; General Procedure A}

$\mathrm{N}$-Protected tryptamine 2 (1.00 equiv) was dissolved in $\mathrm{CH}_{2} \mathrm{Cl}_{2}(2.5$ $\mathrm{mL} / \mathrm{mmol})$ and the solution was cooled to $0{ }^{\circ} \mathrm{C}$. Pyridine $(1.05$ equiv) was added and, subsequently, 2,2,2-trifluoroacetic anhydride (1.05 equiv) was added dropwise. The mixture was allowed to warm to r.t. and stirred for $16 \mathrm{~h}$. After completion, the mixture was washed with aq $2 \mathrm{M} \mathrm{HCl}(3 \times 2 \mathrm{~mL} / \mathrm{mmol})$ and dried over $\mathrm{MgSO}_{4}$. After filtration and removal of all volatiles under reduced pressure, the crude product 3 was obtained, which was used without further purification.

\section{2,2,2- $N$-[2-(1-Methyl-1H-indol-3-yl)ethyl]trifluoroacetamide} (3a)

Obtained according to general procedure A from 2a (1.00 equiv, $3.48 \mathrm{~g}, 20 \mathrm{mmol}$ ).

Yield: $4.70 \mathrm{~g}$ (17.40 mmol, 87\%); brown solid.

${ }^{1} \mathrm{H}$ NMR $\left(201 \mathrm{MHz}, \mathrm{CDCl}_{3}\right): \delta=7.59(\mathrm{~d}, J=7.8 \mathrm{~Hz}, 1 \mathrm{H}), 7.42$ 7.09 (m, $3 \mathrm{H}), 6.91(\mathrm{~s}, 1 \mathrm{H}), 6.70-6.30(\mathrm{br} \mathrm{s}, 1 \mathrm{H}), 3.76(\mathrm{~s}, 3 \mathrm{H}), 3.68$ (dd, $J=12.9,6.5 \mathrm{~Hz}, 2 \mathrm{H}), 3.15-2.97$ (m, $2 \mathrm{H})$.

${ }^{13} \mathrm{C}$ NMR (50 MHz, $\mathrm{CDCl}_{3}$ ): $\delta=137.18,127.37,126.91,121.98$, $119.16,118.50,110.14,109.44,40.28,32.61,24.56 . \mathrm{COCF}_{3}$ peaks not observed.

${ }^{19} \mathrm{~F}$ NMR (189 MHz, $\left.\mathrm{CDCl}_{3}\right): \delta=-76.00$.

HRMS (ESI+): $m / z\left[\mathrm{M}+\mathrm{H}^{+}\right]$calcd for $\mathrm{C}_{13} \mathrm{H}_{14} \mathrm{~F}_{3} \mathrm{~N}_{2} \mathrm{O}: 271.1053$; found: 271.1035 .

\section{$N$-[2-(1-Benzyl-1H-indol-3-yl)ethyl]-2,2,2-trifluoroacetamide (3b)}

Obtained according to general procedure A from $\mathbf{2 b}$ (1.00 equiv, $5.01 \mathrm{~g}, 20 \mathrm{mmol})$.

Yield: $6.44 \mathrm{~g}$ (18.60 mmol, 93\%); brown solid.

${ }^{1} \mathrm{H} \mathrm{NMR}\left(201 \mathrm{MHz}, \mathrm{CDCl}_{3}\right): \delta=7.81-7.59(\mathrm{~m}, 1 \mathrm{H}), 7.51-7.11(\mathrm{~m}$, $8 \mathrm{H}), 7.09-6.90$ (m, $2 \mathrm{H}), 5.31$ (s, $2 \mathrm{H}), 3.73$ (dd, $J=13.1,6.7 \mathrm{~Hz}$, $2 \mathrm{H}), 3.12(\mathrm{t}, J=7.0 \mathrm{~Hz}, 2 \mathrm{H})$.
${ }^{13} \mathrm{C}$ NMR (50 MHz, $\left.\mathrm{CDCl}_{3}\right): \delta=137.24,136.68,128.77,128.60$, $127.58,127.51,126.66,126.13,122.00,119.26,118.59,110.84$, $109.80,49.68,40.04,24.41 . \mathrm{COCF}_{3}$ peaks not observed.

${ }^{19} \mathrm{~F} \mathrm{NMR}\left(189 \mathrm{MHz}, \mathrm{CDCl}_{3}\right): \delta=-75.74$.

HRMS (APCI): $\mathrm{m} / z\left[\mathrm{M}+\mathrm{H}^{+}\right]$calcd for $\mathrm{C}_{19} \mathrm{H}_{18} \mathrm{~F}_{3} \mathrm{~N}_{2} \mathrm{O}: 347.1366$; found: 347.1361 .

$N$-[2-(1-Benzyl-5-methoxy-1H-indol-3-yl)ethyl]-2,2,2-trifluoroacetamide (3c)

Obtained according to general procedure A from 2c (1.00 equiv, $1.475 \mathrm{~g}, 5.26 \mathrm{mmol})$.

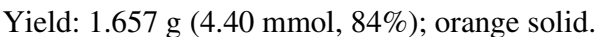

${ }^{1} \mathrm{H}$ NMR (400 MHz, $\left.\mathrm{CDCl}_{3}\right): \delta=7.35-7.22(\mathrm{~m}, 2 \mathrm{H}), 7.18(\mathrm{~d}$, $J=8.9 \mathrm{~Hz}, 1 \mathrm{H}), 7.10(\mathrm{~d}, J=6.5 \mathrm{~Hz}, 2 \mathrm{H}), 7.02(\mathrm{~s}, 1 \mathrm{H}), 6.95(\mathrm{~s}$, $1 \mathrm{H}), 6.91-6.83(\mathrm{~m}, 1 \mathrm{H}), 6.41(\mathrm{br} \mathrm{s}, 1 \mathrm{H}), 5.25$ (s, $2 \mathrm{H}), 3.86$ (s, $3 \mathrm{H}), 3.68(\mathrm{dd}, J=12.7,6.4 \mathrm{~Hz}, 2 \mathrm{H}), 3.02(\mathrm{t}, J=6.6 \mathrm{~Hz}, 2 \mathrm{H})$.

${ }^{13} \mathrm{C}$ NMR $\left(101 \mathrm{MHz}, \mathrm{CDCl}_{3}\right): \delta=154.13,137.31,132.10,128.77$, $127.89,127.69,126.85,126.73,112.47,110.83,110.23,100.32$, $55.85,50.14,40.00,24.64 . \mathrm{COCF}_{3}$ peaks not observed.

${ }^{19} \mathrm{~F} \mathrm{NMR}\left(376 \mathrm{MHz}, \mathrm{CDCl}_{3}\right): \delta=-75.97$.

HRMS (ESI+): $m / z\left[\mathrm{M}+\mathrm{Na}^{+}\right]$calcd for $\mathrm{C}_{20} \mathrm{H}_{19} \mathrm{~F}_{3} \mathrm{~N}_{2} \mathrm{O}_{2} \mathrm{Na}$ : 399.1291; found: 399.1277 .

$\mathrm{N}$-[2-(1H-Indol-3-yl)ethyl]-2,2,2-trifluoroacetamide (4)

Tryptamine (1.00 equiv, $1.00 \mathrm{~g}, 6.24 \mathrm{mmol}$ ) was dissolved in $\mathrm{CH}_{2} \mathrm{Cl}_{2}(50 \mathrm{~mL})$ and the solution was cooled to $0{ }^{\circ} \mathrm{C}$. Pyridine $(0.530 \mathrm{~mL}, 6.55 \mathrm{mmol}, 4.00$ equiv) was added and, subsequently, trifluoroacetic anhydride $(0.926 \mathrm{~mL}, 6.55 \mathrm{mmol}, 5.00$ equiv) was added dropwise. The mixture was allowed to warm to r.t. and stirred for $16 \mathrm{~h}$. After completion (reaction monitored by TLC), the mixture was washed with aq $2 \mathrm{M} \mathrm{HCl}(3 \times 10 \mathrm{~mL})$ and dried over $\mathrm{MgSO}_{4}$, filtered, and all volatiles were removed under reduced pressure to give 4, which was used without further purification.

Yield: $1.567 \mathrm{~g}$ (6.12 mmol, 98\%); brown solid.

${ }^{1} \mathrm{H}$ NMR $\left(201 \mathrm{MHz}, \mathrm{CDCl}_{3}\right): \delta=8.46($ br s, $1 \mathrm{H}), 7.71(\mathrm{~d}$, $J=7.6 \mathrm{~Hz}, 1 \mathrm{H}), 7.51-7.20(\mathrm{~m}, 3 \mathrm{H}), 7.17-6.97(\mathrm{~m}, 2 \mathrm{H}), 3.70$ (q, $J=6.6 \mathrm{~Hz}, 2 \mathrm{H}), 3.10(\mathrm{t}, J=6.9 \mathrm{~Hz}, 2 \mathrm{H})$.

${ }^{13} \mathrm{C} \mathrm{NMR}\left(50 \mathrm{MHz}, \mathrm{CDCl}_{3}\right): \delta=157.40(\mathrm{q}, J=36.9 \mathrm{~Hz}), 136.31$, $126.85,122.28,122.02,119.31,118.19,115.84(\mathrm{q}, J=286.8 \mathrm{~Hz})$, 111.36, 111.29, 40.17, 24.31.

${ }^{19} \mathrm{~F} \mathrm{NMR}\left(189 \mathrm{MHz}, \mathrm{CDCl}_{3}\right): \delta=-75.89$.

HRMS (ESI+): $m / z\left[\mathrm{M}+\mathrm{H}^{+}\right]$calcd for $\mathrm{C}_{12} \mathrm{H}_{12} \mathrm{~F}_{3} \mathrm{~N}_{2} \mathrm{O}: 257.0896$; found: 257.0877 .

\section{Chlorination of 4/3a to give 5; General Procedure B}

Trifluoracetamide $\mathbf{4}$ or 3a (1.00 equiv) was dissolved in $\mathrm{CH}_{2} \mathrm{Cl}_{2}(10$ $\mathrm{mL} / \mathrm{mmol})$ at $21{ }^{\circ} \mathrm{C}$, and a solution of iodine monochloride $(1 \mathrm{~N}$ in $\mathrm{CH}_{2} \mathrm{Cl}_{2}, 1.10$ equiv) was added dropwise. The reaction mixture was stirred at this temperature until full conversion was reached (reaction monitored by TLC). The mixture was washed with $\mathrm{H}_{2} \mathrm{O}$ (10 $\mathrm{mL} / \mathrm{mmol})$ and brine $(10 \mathrm{~mL} / \mathrm{mmol})$, and the organic phases were dried over $\mathrm{MgSO}_{4}$. Removal of all volatiles and purification by column chromatography gave $\mathbf{5}$.

\section{$N$-[2-(2-Chloro-1H-indol-3-yl)ethyl]-2,2,2-trifluoroacetamide} (5a)

Obtained according to general procedure B from 4 (1.00 equiv, 2.00 $\mathrm{g}, 7.81 \mathrm{mmol})$ after purification by column chromatography $\left(\mathrm{SiO}_{2}\right.$; pentane-EtOAc, 10:1).

Yield: $1.807 \mathrm{~g}(6.22 \mathrm{mmol}, 80 \%)$; light-brown solid; $R_{f}=0.75$ (pentane-EtOAc, 8:2). 
${ }^{1} \mathrm{H}$ NMR $\left(201 \mathrm{MHz}, \mathrm{CDCl}_{3}\right): \delta=8.43($ br s, $1 \mathrm{H}), 7.56-7.43(\mathrm{~m}$, $1 \mathrm{H}), 7.38-7.06(\mathrm{~m}, 3 \mathrm{H}), 6.60(\mathrm{br} \mathrm{s}, 1 \mathrm{H}), 3.64(\mathrm{q}, J=6.5 \mathrm{~Hz}, 2 \mathrm{H})$, $3.03(\mathrm{t}, J=6.6 \mathrm{~Hz}, 2 \mathrm{H})$.

${ }^{13} \mathrm{C}$ NMR $\left(50 \mathrm{MHz}, \mathrm{CDCl}_{3}\right): \delta=134.49,127.08,122.64,121.71$, $120.46,117.68,110.75,107.79,39.77,23.15 . \mathrm{COCF}_{3}$ resonances not observed.

${ }^{19} \mathrm{~F} \mathrm{NMR}\left(189 \mathrm{MHz}, \mathrm{CDCl}_{3}\right): \delta=-76.06$.

HRMS (APCI): $m / z,\left[\mathrm{M}-\mathrm{Cl}^{-}\right]$calcd for $\mathrm{C}_{12} \mathrm{H}_{10} \mathrm{~F}_{3} \mathrm{~N}_{2} \mathrm{O}: 255.0745$; found: 255.0737 .

\section{$N$-[2-(2-Chloro-1-methyl-1H-indol-3-yl)ethyl]-2,2,2-trifluoro- acetamide (5b)}

Obtained according to general procedure B from 3a (1.00 equiv, $1.920 \mathrm{~g}, 7.10 \mathrm{mmol}$ ) after purification by column chromatography $\left(\mathrm{SiO}_{2}\right.$; pentane-EtOAc, 10:1).

Yield: $1.688 \mathrm{~g}$ (5.54 mmol, 78\%); yellow solid; $R_{f}=0.80$ (pentaneEtOAc, 8:2).

${ }^{1} \mathrm{H}$ NMR $\left(201 \mathrm{MHz}, \mathrm{CDCl}_{3}\right): \delta=7.52(\mathrm{~d}, J=7.6 \mathrm{~Hz}, 1 \mathrm{H}), 7.35$ $7.25(\mathrm{~m}, 2 \mathrm{H}), 7.24-7.08(\mathrm{~m}, 1 \mathrm{H}), 6.48(\mathrm{br} \mathrm{s}, 1 \mathrm{H}), 3.74(\mathrm{~d}$, $J=2.5 \mathrm{~Hz}, 3 \mathrm{H}), 3.64(\mathrm{q}, J=6.4 \mathrm{~Hz}, 2 \mathrm{H}), 3.06(\mathrm{t}, J=6.6 \mathrm{~Hz}, 2 \mathrm{H})$.

${ }^{13} \mathrm{C}$ NMR $\left(50 \mathrm{MHz}, \mathrm{CDCl}_{3}\right): \delta=135.82,126.20,124.50,122.22$, $120.19,117.70,109.30,106.86,39.87,29.89,23.58 . \mathrm{COCF}_{3}$ resonances not observed.

${ }^{19} \mathrm{~F}$ NMR $\left(189 \mathrm{MHz}, \mathrm{CDCl}_{3}\right): \delta=-76.06$

HRMS (APCI): $m / z$ [M $\left.-\mathrm{Cl}^{-}\right]$calcd for $\mathrm{C}_{13} \mathrm{H}_{12} \mathrm{~F}_{3} \mathrm{~N}_{2} \mathrm{O}: 269.0902$; found: 269.0894 .

\section{Iodination of 3 To Give 7; General Procedure C}

Trifluoroacetamide 3 (1.00 equiv) was dissolved in $\mathrm{Et}_{2} \mathrm{O}(5 \mathrm{~mL} /$ mmol) and cooled to $0{ }^{\circ} \mathrm{C}$. BuLi (1.6 M in hexanes, 2.50 equiv) was added dropwise and the reaction mixture was allowed to warm to r.t. After $2 \mathrm{~h}$, the reaction mixture was cooled to $0{ }^{\circ} \mathrm{C}$, and iodine $(1.50$ equiv) was added. After warming to r.t., the reaction was quenched by addition of sat. aq $\mathrm{Na}_{2} \mathrm{~S}_{2} \mathrm{O}_{3}(5 \mathrm{~mL} / \mathrm{mmol})$, washed with $\mathrm{H}_{2} \mathrm{O}$ $(3 \times 5 \mathrm{~mL} / \mathrm{mmol})$ and extracted with $\mathrm{Et}_{2} \mathrm{O}(3 \times 5 \mathrm{~mL} / \mathrm{mmol})$. After drying over $\mathrm{MgSO}_{4}$, all volatiles were removed under reduced pressure. Purification of the crude mixture by column chromatography gave the desired products 7 .

\section{2,2,2-Trifluoro- $N$-[2-(2-iodo-1-methyl-1 $H$-indol-3-yl)eth- yl]acetamide (7a)}

Obtained according to general procedure $\mathrm{C}$ from 3a (1.00 equiv, $0.500 \mathrm{~g}, 1.850 \mathrm{mmol}$ ) and purified by column chromatography $\left(\mathrm{SiO}_{2}\right.$; pentane-EtOAc, 8:2).

Yield: $0.403 \mathrm{~g}(1.018 \mathrm{mmol}, 55 \%)$; white solid; $R_{f}=0.80$ (pentaneEtOAc, 8:2).

${ }^{1} \mathrm{H}$ NMR $\left(201 \mathrm{MHz}, \mathrm{CDCl}_{3}\right): \delta=7.60(\mathrm{~d}, J=7.6 \mathrm{~Hz}, 1 \mathrm{H}), 7.32(\mathrm{t}$, $J=8.3 \mathrm{~Hz}, 1 \mathrm{H}), 7.18(\mathrm{dd}, J=15.9,7.9 \mathrm{~Hz}, 2 \mathrm{H}), 7.07$ (br s, $1 \mathrm{H})$, $3.75(\mathrm{~s}, 3 \mathrm{H}), 3.65$ (q, $J=6.7 \mathrm{~Hz}, 2 \mathrm{H}), 3.09(\mathrm{t}, J=6.9 \mathrm{~Hz}, 2 \mathrm{H})$.

${ }^{13} \mathrm{C}$ NMR $\left(50 \mathrm{MHz}, \mathrm{CDCl}_{3}\right): \delta=159.29$ (q, $J=36.8 \mathrm{~Hz}$ ), 138.40 , $127.36,122.03,119.50,117.49,116.63,115.76(\mathrm{q}, J=288.6 \mathrm{~Hz})$, $109.69,87.84,39.92,33.99,26.61$.

${ }^{19} \mathrm{~F} \mathrm{NMR}\left(189 \mathrm{MHz}, \mathrm{CDCl}_{3}\right): \delta=-75.67$.

HRMS (APCI): $m / z\left[\mathrm{M}+\mathrm{H}^{+}-\mathrm{I}\right]$ calcd for $\mathrm{C}_{13} \mathrm{H}_{13} \mathrm{~F}_{3} \mathrm{~N}_{2} \mathrm{O}: 270.0980$; found: 269.9763

\section{$N$-[2-(1-Benzyl-2-iodo-1H-indol-3-yl)ethyl]-2,2,2-trifluoro-} acetamide (7b)

Obtained according to general procedure $\mathrm{C}$ from $3 \mathbf{b}$ (1.00 equiv, $2.00 \mathrm{~g}, 5.77 \mathrm{mmol})$ and purified by column chromatography $\left(\mathrm{SiO}_{2}\right.$; pentane-EtOAc, 10:1).
Yield: $1.309 \mathrm{~g}(2.77 \mathrm{mmol}, 48 \%)$; white solid; $R_{f}=0.66$ (pentaneEtOAc, 10:1).

${ }^{1} \mathrm{H} \mathrm{NMR}\left(201 \mathrm{MHz}, \mathrm{CDCl}_{3}\right): \delta=7.64-7.51(\mathrm{~m}, 1 \mathrm{H}), 7.37-7.21(\mathrm{~m}$, 4 H), 7.20-7.09 (m, 2 H), 7.07-6.97 (m, 2 H), 6.35 (br s, 1 H), 5.44 (s, $2 \mathrm{H}), 3.69$ (q, $J=6.4 \mathrm{~Hz}, 2 \mathrm{H}), 3.11(\mathrm{t}, J=6.6 \mathrm{~Hz}, 2 \mathrm{H})$.

${ }^{13} \mathrm{C}$ NMR $\left(101 \mathrm{MHz}, \mathrm{CDCl}_{3}\right): \delta=157.21$ (q, $\left.J=36.4 \mathrm{~Hz}\right), 138.37$, $136.87,128.65,127.78,127.44,126.24,122.48,119.99,117.74$, 117.44, $115.75(\mathrm{q}, J=286.8 \mathrm{~Hz}), 110.34,87.87,50.60,39.87$, 26.83.

${ }^{19} \mathrm{~F}$ NMR $\left(376 \mathrm{MHz}, \mathrm{CDCl}_{3}\right): \delta=-75.77$.

HRMS (APCI): $m / z\left[\mathrm{M}+\mathrm{H}^{+}-\mathrm{I}\right]$ calcd for $\mathrm{C}_{19} \mathrm{H}_{17} \mathrm{~F}_{3} \mathrm{~N}_{2} \mathrm{O}: 346.1293$; found: 346.0073 .

\section{$N$-[2-(1-Benzyl-2-iodo-5-methoxy-1H-indol-3-yl)ethyl]-2,2,2-} trifluoroacetamide $(7 \mathrm{c})$

Obtained according to general procedure $\mathrm{C}$ from $3 \mathbf{c}$ (1.00 equiv, $1.657 \mathrm{~g}, 4.40 \mathrm{mmol})$ and purified by column chromatography $\left(\mathrm{SiO}_{2}\right.$; pentane-EtOAc, 10:1).

Yield: $1.150 \mathrm{~g}$ ( $2.289 \mathrm{mmol}, 52 \%)$; white solid; $R_{f}=0.55$ (pentaneEtOAc, 10:1).

${ }^{1} \mathrm{H}$ NMR $\left(201 \mathrm{MHz}, \mathrm{CDCl}_{3}\right): \delta=7.43-7.21(\mathrm{~m}, 4 \mathrm{H}), 7.15(\mathrm{~d}$, $J=8.9 \mathrm{~Hz}, 1 \mathrm{H}), 7.01(\mathrm{dd}, J=9.3,2.5 \mathrm{~Hz}, 2 \mathrm{H}), 6.78(\mathrm{dd}, J=8.9$, $2.4 \mathrm{~Hz}, 1 \mathrm{H}), 6.37$ (br s, $1 \mathrm{H}), 5.39$ (s, $2 \mathrm{H}), 3.84$ (s, $3 \mathrm{H}), 3.68$ (dd, $J=12.7,6.4 \mathrm{~Hz}, 2 \mathrm{H}), 3.07(\mathrm{t}, J=6.6 \mathrm{~Hz}, 2 \mathrm{H})$.

${ }^{13} \mathrm{C}$ NMR $\left(50 \mathrm{MHz}, \mathrm{CDCl}_{3}\right): \delta=154.41,137.00,133.78,128.76$, $128.56,127.55,126.98,126.27,116.70,112.81,111.30,99.36$, $55.79,50.92,39.88,26.88 . \mathrm{COCF}_{3}$ resonances not observed.

${ }^{19} \mathrm{~F} \mathrm{NMR}\left(189 \mathrm{MHz}, \mathrm{CDCl}_{3}\right): \delta=-75.88$.

HRMS (ESI+): $m / z\left[\mathrm{M}+\mathrm{Na}^{+}\right]$calcd for $\mathrm{C}_{20} \mathrm{H}_{18} \mathrm{~F}_{3} \mathrm{IN}_{2} \mathrm{O}_{2} \mathrm{Na}$ : 525.0257; found: 525.0236 .

\section{Stille Reaction of 7 To Give Allylic Alcohols 8; General Proce- dure D}

Iodide 7 (1.00 equiv), (E)-3-(tributylstannyl)prop-2-en-1-ol (6; 1.50 equiv), bis(triphenylphosphine)palladium(II) chloride (5.0 mol\%) and $\mathrm{LiCl}$ (3.0 equiv) were dissolved in DMF $(20 \mathrm{~mL} / \mathrm{mmol})$ and the mixture was heated to $75^{\circ} \mathrm{C}$ for $16 \mathrm{~h}$. The reaction was quenched by addition of $\mathrm{H}_{2} \mathrm{O}(20 \mathrm{~mL} / \mathrm{mmol})$, EtOAc $(20 \mathrm{~mL} / \mathrm{mmol})$ was added, and the organic phases were washed with brine $(20 \mathrm{~mL} / \mathrm{mmol})$. After drying over $\mathrm{MgSO}_{4}$ and removal of all volatiles, the crude product was purified by column chromatography to give $\mathbf{8}$.

(E)-2,2,2-Trifluoro- $N$-\{2-[2-(3-hydroxyprop-1-enyl)-1-methyl$1 \mathrm{H}$-indol-3-yl]ethyl $\}$ acetamide $(8 \mathrm{a})$

Obtained according to general procedure D from 7a (1.00 equiv, $0.300 \mathrm{~g}, 0.757 \mathrm{mmol}$ ) and purified by column chromatography $\left(\mathrm{SiO}_{2}\right.$; pentane-EtOAc, 1:1).

Yield: $0.167 \mathrm{~g}(0.512 \mathrm{mmol}, 68 \%)$; orange solid; $R_{f}=0.30$ (pentane-EtOAc, 1:1).

${ }^{1} \mathrm{H}$ NMR $\left(201 \mathrm{MHz}, \mathrm{CDCl}_{3}\right): \delta=7.58(\mathrm{dd}, J=7.8,0.7 \mathrm{~Hz}, 1 \mathrm{H})$, 7.44-7.22 (m, $3 \mathrm{H}), 7.20-7.07$ (m, $1 \mathrm{H}), 6.69(\mathrm{~d}, J=16.3 \mathrm{~Hz}, 1 \mathrm{H})$, $6.29(\mathrm{dd}, J=13.4,8.1 \mathrm{~Hz}, 1 \mathrm{H}), 4.40(\mathrm{~d}, J=5.3 \mathrm{~Hz}, 2 \mathrm{H}), 3.69(\mathrm{~s}$, $3 \mathrm{H}), 3.59$ (dd, $J=13.7,6.8 \mathrm{~Hz}, 2 \mathrm{H}), 3.14(\mathrm{t}, J=7.3 \mathrm{~Hz}, 2 \mathrm{H})$.

${ }^{13} \mathrm{C}$ NMR $\left(50 \mathrm{MHz}, \mathrm{CDCl}_{3}\right): \delta=157.44(\mathrm{q}, J=37.3 \mathrm{~Hz}), 137.16$, 134.46, 133.84, 127.26, 122.17, 119.43, 118.92, 118.09, 115.78 (q, $J=287.9 \mathrm{~Hz}), 109.19,109.09,63.31,40.45,30.42,23.92$.

${ }^{19} \mathrm{~F} \mathrm{NMR}\left(189 \mathrm{MHz}, \mathrm{CDCl}_{3}\right): \delta=-75.88$.

HRMS (APCI+): $m / z\left[\mathrm{M}+\mathrm{H}^{+}\right]$calcd for $\mathrm{C}_{16} \mathrm{H}_{18} \mathrm{~F}_{3} \mathrm{~N}_{2} \mathrm{O}_{2}: 327.1320$; found: 325.1147 . 
(E)- $N$-\{2-[1-Benzyl-2-(3-hydroxyprop-1-enyl)-1H-indol-3yl]ethyl\}-2,2,2-trifluoroacetamide (8b)

Obtained according to general procedure D from $7 \mathbf{b}$ (1.00 equiv, $0.285 \mathrm{~g}, 0.604 \mathrm{mmol}$ ) and purified by column chromatography $\left(\mathrm{SiO}_{2}\right.$; pentane-EtOAc, 1:1).

Yield: $0.172 \mathrm{~g}(0.427 \mathrm{mmol}, 71 \%)$; white solid; $R_{f}=0.50$ (pentaneEtOAc, 1:1).

${ }^{1} \mathrm{H}$ NMR $\left(400 \mathrm{MHz}, \mathrm{CDCl}_{3}\right): \delta=7.59(\mathrm{~d}, J=7.7 \mathrm{~Hz}, 1 \mathrm{H}), 7.33$ $7.10(\mathrm{~m}, 6 \mathrm{H}), 7.00(\mathrm{~d}, J=7.0 \mathrm{~Hz}, 2 \mathrm{H}), 6.71-6.54(\mathrm{~m}, 2 \mathrm{H}), 6.18$ (dt, $J=16.1,5.3 \mathrm{~Hz}, 1 \mathrm{H}), 5.37(\mathrm{~s}, 2 \mathrm{H}), 4.28(\mathrm{~d}, J=5.2 \mathrm{~Hz}, 2 \mathrm{H})$, $3.65(\mathrm{dd}, J=13.4,6.8 \mathrm{~Hz}, 2 \mathrm{H}), 3.19(\mathrm{t}, J=7.1 \mathrm{~Hz}, 2 \mathrm{H})$.

${ }^{13} \mathrm{C}$ NMR (101 MHz, $\left.\mathrm{CDCl}_{3}\right): \delta=137.61,137.15,134.73,134.59$, $128.80,127.56,127.37,125.87,122.68,119.98,118.80,118.33$, $109.88,109.60,63.47,47.31,40.42,24.04 . \mathrm{COCF}_{3}$ resonances not observed.

${ }^{19} \mathrm{~F}$ NMR $\left(376 \mathrm{MHz}, \mathrm{CDCl}_{3}\right): \delta=-76.00$.

HRMS (ESI+): $m / z\left[\mathrm{M}+\mathrm{Na}^{+}\right]$calcd for $\mathrm{C}_{22} \mathrm{H}_{21} \mathrm{~F}_{3} \mathrm{~N}_{2} \mathrm{O}_{2} \mathrm{Na}$ : 425.1447; found: 425.1432 .

(E)- $N$-\{2-[1-Benzyl-2-(3-hydroxyprop-1-en-1-yl)-5-methoxy$1 H$-indol-3-yl]ethyl\}-2,2,2-trifluoroacetamide (8c)

Obtained according to general procedure D from $7 \mathbf{c}$ (1.00 equiv, $0.270 \mathrm{~g}, 0.538 \mathrm{mmol}$ ) and purified by column chromatography $\left(\mathrm{SiO}_{2}\right.$; pentane-EtOAc, 1:1).

Yield: $0.158 \mathrm{~g}(0.366 \mathrm{mmol}, 68 \%)$; white solid; $R_{f}=0.5$ (pentaneEtOAc, 1:1).

${ }^{1} \mathrm{H}$ NMR $\left(400 \mathrm{MHz}, \mathrm{CDCl}_{3}\right): \delta=7.26(\mathrm{~d}, J=6.7 \mathrm{~Hz}, 3 \mathrm{H}), 7.08(\mathrm{~d}$, $J=8.6 \mathrm{~Hz}, 1 \mathrm{H}), 7.03(\mathrm{~s}, 1 \mathrm{H}), 6.98(\mathrm{~d}, J=6.0 \mathrm{~Hz}, 2 \mathrm{H}), 6.91(\mathrm{br} \mathrm{s}$, $1 \mathrm{H}), 6.83(\mathrm{~d}, J=8.2 \mathrm{~Hz}, 1 \mathrm{H}), 6.58(\mathrm{~d}, J=16.2 \mathrm{~Hz}, 1 \mathrm{H}), 6.15(\mathrm{~d}$, $J=15.8 \mathrm{~Hz}, 1 \mathrm{H}), 5.31(\mathrm{~s}, 2 \mathrm{H}), 4.25(\mathrm{~s}, 2 \mathrm{H}), 3.85(\mathrm{~s}, 3 \mathrm{H}), 3.62(\mathrm{~d}$, $J=5.6 \mathrm{~Hz}, 2 \mathrm{H}), 3.14($ br s, $2 \mathrm{H})$.

${ }^{13} \mathrm{C} \mathrm{NMR}\left(101 \mathrm{MHz}, \mathrm{CDCl}_{3}\right): \delta=157.38(\mathrm{q}, J=37.6 \mathrm{~Hz}), 154.40$, $137.70,135.12,134.11,132.39,128.75,127.92,127.30,125.80$, $118.89,115.83(\mathrm{q}, J=287.4 \mathrm{~Hz}), 112.75,110.70,109.22,100.08$, $63.40,55.83,47.34,40.29,24.06$.

${ }^{19} \mathrm{~F} \mathrm{NMR}\left(376 \mathrm{MHz}, \mathrm{CDCl}_{3}\right): \delta=-75.94$.

HRMS (ESI+): $m / z\left[\mathrm{M}+\mathrm{Na}^{+}\right]$calcd for $\mathrm{C}_{23} \mathrm{H}_{23} \mathrm{~F}_{3} \mathrm{~N}_{2} \mathrm{O}_{3} \mathrm{Na}$ : 455.1553; found: 455.1539 .

\section{Conversion of Allylic Alcohols 8 into Allylic Carbonates 9; General Procedure E}

The appropriate allylic alcohol 8 (1.00 equiv) and anhydrous pyridine (1.50 equiv) were dissolved in $\mathrm{CH}_{2} \mathrm{Cl}_{2}(2 \mathrm{~mL} / \mathrm{mmol})$ and the reaction mixture was cooled to $0{ }^{\circ} \mathrm{C}$. Methyl chloroformate $(1.10$ equiv) was added dropwise and the reaction mixture was stirred for $16 \mathrm{~h}$, while allowing it to warm to $21^{\circ} \mathrm{C}$. When TLC indicated full conversion of the starting material, the mixture was washed with aq $2 \mathrm{M} \mathrm{HCl}(3 \times 2 \mathrm{~mL} / \mathrm{mmol})$ and dried over $\mathrm{MgSO}_{4}$. After filtration and removal of all volatiles under reduced pressure, the desired products 9 were obtained in sufficient purity.

(E)-Methyl 3-\{1-Methyl-3-[2-(2,2,2-trifluoroacetamido)ethyl]$1 \mathrm{H}$-indol-2-yl $\}$ allyl Carbonate (9a)

Obtained according to general procedure $\mathrm{E}$ from 8a (1.00 equiv, $0.160 \mathrm{~g}, 0.490 \mathrm{mmol})$.

Yield: $0.185 \mathrm{~g}(0.481 \mathrm{mmol}, 98 \%)$; yellow foam.

${ }^{1} \mathrm{H} \mathrm{NMR}\left(400 \mathrm{MHz}, \mathrm{CDCl}_{3}\right): \delta=7.62-7.55(\mathrm{~m}, 1 \mathrm{H}), 7.32-7.25(\mathrm{~m}$, $2 \mathrm{H}), 7.17-7.10(\mathrm{~m}, 1 \mathrm{H}), 6.84-6.72(\mathrm{~m}, 2 \mathrm{H}), 6.19$ (dt, $J=16.2$, $6.2 \mathrm{~Hz}, 1 \mathrm{H}), 4.85(\mathrm{dd}, J=6.2,1.4 \mathrm{~Hz}, 2 \mathrm{H}), 3.83-3.82(\mathrm{~s}, 2 \mathrm{H})$, 3.73 (s, $3 \mathrm{H}), 3.60$ (q, $J=6.8 \mathrm{~Hz}, 2 \mathrm{H}), 3.13$ (t, $J=7.1 \mathrm{~Hz}, 2 \mathrm{H})$.

${ }^{13} \mathrm{C} \mathrm{NMR}\left(101 \mathrm{MHz}, \mathrm{CDCl}_{3}\right): \delta=157.14(\mathrm{q}, J=36.5 \mathrm{~Hz}), 155.47$, $137.51,133.42$, 127.18, 126.88, 122.84, 122.70, 119.66, 118.37, $115.73(\mathrm{q}, J=288.2 \mathrm{~Hz}), 110.40,109.30,68.29,54.78,40.41$, 30.70, 23.96.

${ }^{19} \mathrm{~F} \mathrm{NMR}\left(376 \mathrm{MHz}, \mathrm{CDCl}_{3}\right): \delta=-75.98$.

HRMS (APCI): $m / z\left[\mathrm{M}-\mathrm{OCO}_{2} \mathrm{Me}\right]$ calcd for $\mathrm{C}_{16} \mathrm{H}_{16} \mathrm{~F}_{3} \mathrm{~N}_{2} \mathrm{O}$ : 309.1215; found: 309.1199 .

(E)-3-\{1-Benzyl-3-[2-(2,2,2-trifluoroacetamido)ethyl]-1 $H$-indol-2-yl \}allyl Methyl Carbonate (9b)

Obtained according to general procedure E from $\mathbf{8 b}$ (1.00 equiv, $0.230 \mathrm{~g}, 0.572 \mathrm{mmol})$.

Yield: $0.257 \mathrm{~g}(0.558 \mathrm{mmol}, 98 \%)$; yellow foam.

${ }^{1} \mathrm{H}$ NMR $\left(201 \mathrm{MHz}, \mathrm{CDCl}_{3}\right): \delta=7.65(\mathrm{~d}, J=6.9 \mathrm{~Hz}, 1 \mathrm{H}), 7.41-$ $7.10(\mathrm{~m}, 6 \mathrm{H}), 7.09-6.98(\mathrm{~m}, 2 \mathrm{H}), 7.10-6.95(\mathrm{~m}, 2 \mathrm{H}), 6.12(\mathrm{dt}$, $J=16.2,6.1 \mathrm{~Hz}, 1 \mathrm{H}), 5.38(\mathrm{~s}, 2 \mathrm{H}), 4.76(\mathrm{~d}, J=6.1 \mathrm{~Hz}, 2 \mathrm{H}), 3.79$ (s, $3 \mathrm{H}), 3.66$ (dd, $J=13.2,6.7 \mathrm{~Hz}, 2 \mathrm{H}), 3.19$ (t, $J=7.0 \mathrm{~Hz}, 2 \mathrm{H})$.

${ }^{13} \mathrm{C} \mathrm{NMR}\left(50 \mathrm{MHz}, \mathrm{CDCl}_{3}\right): \delta=155.39,137.39,137.36,133.54$, $128.74,127.53,127.47,127.34,125.82,123.01,122.57,120.03$, $118.55,110.79,109.83,68.12,54.76,47.29,40.33,24.10 . \mathrm{COCF}_{3}$ resonances not observed.

${ }^{19} \mathrm{~F}$ NMR $\left(189 \mathrm{MHz}, \mathrm{CDCl}_{3}\right): \delta=-75.95$.

HRMS (APCI): $m / z\left[\mathrm{M}-\mathrm{OCO}_{2} \mathrm{Me}\right]$ calcd for $\mathrm{C}_{22} \mathrm{H}_{20} \mathrm{~F}_{3} \mathrm{~N}_{2} \mathrm{O}$ : 385.1528; found: 385.1507 .

(E)-3-\{1-Benzyl-5-methoxy-3-[2-(2,2,2-trifluoroacetamido)ethyl]-1H-indol-2-yl \}allyl Methyl Carbonate (9c)

Obtained according to general procedure $\mathrm{E}$ from $8 \mathrm{c}$ (1.00 equiv, $0.137 \mathrm{~g}, 0.317 \mathrm{mmol})$.

Yield: $0.142 \mathrm{~g}(0.290 \mathrm{mmol}, 91 \%)$; yellow foam.

${ }^{1} \mathrm{H}$ NMR (201 MHz, $\left.\mathrm{CDCl}_{3}\right): \delta=7.35-7.20(\mathrm{~m}, 3 \mathrm{H}), 7.11(\mathrm{~d}$, $J=8.9 \mathrm{~Hz}, 1 \mathrm{H}), 7.05(\mathrm{~d}, J=2.3 \mathrm{~Hz}, 1 \mathrm{H}), 7.03-6.96(\mathrm{~m}, 2 \mathrm{H}), 6.85$ $(\mathrm{dd}, J=8.9,2.4 \mathrm{~Hz}, 1 \mathrm{H}), 6.79-66.0(\mathrm{~m}, 2 \mathrm{H}), 6.07$ (dt, $J=16.2$, $6.1 \mathrm{~Hz}, 1 \mathrm{H}), 5.33(\mathrm{~s}, 2 \mathrm{H}), 4.74(\mathrm{dd}, J=6.1,1.2 \mathrm{~Hz}, 2 \mathrm{H}), 3.85$ (s, $3 \mathrm{H}), 3.77(\mathrm{~s}, 3 \mathrm{H}), 3.65(\mathrm{dd}, J=13.2,6.7 \mathrm{~Hz}, 2 \mathrm{H}), 3.14(\mathrm{t}$, $J=7.0 \mathrm{~Hz}, 2 \mathrm{H})$.

${ }^{13} \mathrm{C} \mathrm{NMR}\left(50 \mathrm{MHz}, \mathrm{CDCl}_{3}\right): \delta=157.15$ (q, $\left.J=37.0 \mathrm{~Hz}\right), 155.41$, $154.49,137.48,133.95,132.65,128.75,127.82,127.34,127.08$, $125.79,122.74,115.76(\mathrm{q}, J=287.2 \mathrm{~Hz}), 113.38,110.74,110.39$, $100.00,68.18,55.70,54.78,47.41,40.24,24.14$.

${ }^{19} \mathrm{~F} \mathrm{NMR}\left(189 \mathrm{MHz}, \mathrm{CDCl}_{3}\right): \delta=-75.93$.

HRMS (ESI+): $m / z\left[\mathrm{M}+\mathrm{Na}^{+}\right]$calcd for $\mathrm{C}_{25} \mathrm{H}_{25} \mathrm{~F}_{3} \mathrm{~N}_{2} \mathrm{O}_{5} \mathrm{Na}$ : 513.1608; found: 513.1585 .

\section{Allylic Amidation of 9 To Give $\beta$-Carbolines 10; General Proce- dure $\mathbf{F}$}

The appropriate allylic carbonate $\mathbf{9}$ (1.00 equiv) was dissolved in dioxane $(50 \mathrm{~mL} / \mathrm{mmol})$ and $\mathrm{Cs}_{2} \mathrm{CO}_{3}$ (2.00 equiv) was added. The reaction was stirred for $16 \mathrm{~h}$ at $100{ }^{\circ} \mathrm{C}$. After cooling, $\mathrm{H}_{2} \mathrm{O}(50 \mathrm{~mL} /$ $\mathrm{mmol})$ was added and the mixture was extracted with $\mathrm{Et}_{2} \mathrm{O}(3 \times 20$ $\mathrm{mL} / \mathrm{mmol}$ ). After drying over $\mathrm{MgSO}_{4}$ and removal of all volatiles under reduced pressure, the crude product was purified by column chromatography to give the desired products $\mathbf{1 0}$.

\section{2,2,2-Trifluoro-1-[9-methyl-1-vinyl-3,4-dihydro-1H-pyrido-} indol-2(9H)-yl]ethanone (10a)

Obtained according to general procedure $\mathrm{F}$ from 9a (1.00 equiv, $0.020 \mathrm{~g}, 0.052 \mathrm{mmol}$ ) and purified by column chromatography $\left(\mathrm{SiO}_{2}\right.$; pentane-EtOAc, 10:1). The product was isolated as a mixture of two isomers (ratio 1:7).

Yield: $0.013 \mathrm{~g}(0.043 \mathrm{mmol}, 82 \%)$; white solid; $R_{f}=0.95$ (pentaneEtOAc, 10:1).

${ }^{1} \mathrm{H}$ NMR $\left(201 \mathrm{MHz}, \mathrm{CDCl}_{3}\right): \delta$ (major isomer) $=7.57-7.45(\mathrm{~m}$, $1 \mathrm{H}), 7.38-7.23(\mathrm{~m}, 2 \mathrm{H}), 7.23-7.06(\mathrm{~m}, 1 \mathrm{H}), 6.23$ (br d, 
$J=5.2 \mathrm{~Hz}, 1 \mathrm{H}), 6.17-5.95(\mathrm{~m}, 1 \mathrm{H}), 5.48(\mathrm{~d}, J=10.0 \mathrm{~Hz}, 1 \mathrm{H})$, $5.09(\mathrm{~d}, J=17.0 \mathrm{~Hz}, 1 \mathrm{H}), 4.17(\mathrm{br} \mathrm{d}, J=14.1 \mathrm{~Hz}, 1 \mathrm{H}), 3.71-3.47$ (m, $4 \mathrm{H}), 3.12-2.74(\mathrm{~m}, 2 \mathrm{H})$.

${ }^{13} \mathrm{C} \mathrm{NMR}\left(50 \mathrm{MHz}, \mathrm{CDCl}_{3}\right): \delta=133.05,130.56,129.98,125.93$, $122.02,121.22,119.47,118.26,109.07,107.83,51.60,39.91$, 29.86, 22.15. $\mathrm{COCF}_{3}$ resonances not observed.

${ }^{19} \mathrm{~F} \mathrm{NMR}$ (189 MHz, $\mathrm{CDCl}_{3}$ ): $\delta=-68.55$ (minor), -68.99 (major). HRMS (APCI): $m / z\left[\mathrm{M}+\mathrm{H}^{+}\right]$calcd for $\mathrm{C}_{16} \mathrm{H}_{16} \mathrm{~F}_{3} \mathrm{~N}_{2} \mathrm{O}: 309.1209$; found: 309.1220 .

1-\{9-Benzyl-1-vinyl-3,4-dihydro-1H-pyrido[3,4-b]indol-2(9H)yl\}-2,2,2-trifluoroethanone (10b)

Obtained according to general procedure $\mathrm{F}$ from $\mathbf{9 b}$ (1.00 equiv, $0.037 \mathrm{~g}, 0.080 \mathrm{mmol}$ ) and purified by column chromatography $\left(\mathrm{SiO}_{2}\right.$; pentane-EtOAc, 10:1). NMR analysis indicated the presence four isomers in a ratio 1.5:5:1.5:1. When investigated by variabletemperature ${ }^{19} \mathrm{~F}$ NMR spectroscopy, two of the observed resonances coalesce at $80{ }^{\circ} \mathrm{C}$, while one of the other set of two resonances disappeared.

Yield: $0.023 \mathrm{~g}$ ( $0.061 \mathrm{mmol}, 76 \%)$; white solid; $R_{f}=0.85$ (pentaneEtOAc, 10:1).

${ }^{1} \mathrm{H}$ NMR $\left(201 \mathrm{MHz}, \mathrm{CDCl}_{3}\right): \delta$ (major rotamers) $=7.53-7.41(\mathrm{~m}$, $1 \mathrm{H}), 7.31-7.00(\mathrm{~m}, 7 \mathrm{H}), 6.95-6.83(\mathrm{~m}, 1 \mathrm{H}), 6.10-1.03(\mathrm{~m}, 1 \mathrm{H})$, 6.01-5.81 (m, $1 \mathrm{H}), 5.45-5.25(\mathrm{~m}, 2 \mathrm{H}), 5.18(\mathrm{~d}, J=10.3 \mathrm{~Hz}, 1 \mathrm{H})$, 5.13-4.87 (m, $1 \mathrm{H}), 4.20-4.00(\mathrm{~m}, 1 \mathrm{H}), 3.63-3.42(\mathrm{~m}, 1 \mathrm{H}), 3.08-$ $2.72(\mathrm{~m}, 2 \mathrm{H})$.

${ }^{13} \mathrm{C} \mathrm{NMR}\left(50 \mathrm{MHz}, \mathrm{CDCl}_{3}\right): \delta$ (major rotamers) $=137.19,136.86$, $132.94,128.99,128.84,127.54,126.08,125.78,122.34,121.24$, $120.29,119.74,118.34,109.96,47.01,29.70,22.18 . \mathrm{COCF}_{3}$ resonances not observed.

${ }^{19} \mathrm{~F}$ NMR $\left(189 \mathrm{MHz}, \mathrm{CDCl}_{3}\right): \delta=-68.91(1.5),-68.97(5),-69.04$ (1.5), -70.17 (1).

HRMS (ESI+): $m / z\left[\mathrm{M}+\mathrm{H}^{+}\right]$calcd for $\mathrm{C}_{22} \mathrm{H}_{20} \mathrm{~F}_{3} \mathrm{~N}_{2} \mathrm{O}: 385.1522$; found: 385.1511 .

\section{1-\{9-Benzyl-6-methoxy-1-vinyl-3,4-dihydro-1H-pyrido[3,4-} $b$ ]indol-2(9H)-yl\}-2,2,2-trifluoroethanone (10c)

Obtained according to general procedure $\mathrm{F}$ from $9 \mathrm{c}$ (1.00 equiv, $0.025 \mathrm{~g}, 0.051 \mathrm{mmol}$ ) and purified by column chromatography $\left(\mathrm{SiO}_{2}\right.$; pentane-EtOAc, 10:1). NMR analysis indicated the presence four isomers in a ratio 2:6:2:1. See also comment to $\mathbf{1 0 b}$.

Yield: $0.017 \mathrm{~g}(0.042 \mathrm{mmol}, 82 \%)$; white solid; $R_{f}=0.65$ (pentaneEtOAc, 10:1).

${ }^{1} \mathrm{H}$ NMR $\left(400 \mathrm{MHz}, \mathrm{CDCl}_{3}\right): \delta$ (major peaks only) $=7.44-6.77(\mathrm{~m}$, $8 \mathrm{H}), 6.11(\mathrm{~d}, J=5.4 \mathrm{~Hz}, 1 \mathrm{H}), 6.07-5.93(\mathrm{~m}, 1 \mathrm{H}), 5.50-4.98(\mathrm{~m}$, $4 \mathrm{H}), 4.25-4.12$ (m, $1 \mathrm{H}), 3.85$ (s, $3 \mathrm{H}), 3.67-3.54$ (m, $1 \mathrm{H}), 3.11-$ $2.94(\mathrm{~m}, 1 \mathrm{H}), 2.93-2.78(\mathrm{~m}, 1 \mathrm{H})$.

${ }^{13} \mathrm{C} \mathrm{NMR}\left(101 \mathrm{MHz}, \mathrm{CDCl}_{3}\right): \delta=155.97(\mathrm{q}, J=35.2 \mathrm{~Hz}), 154.29$, 136.96, 132.96, 131.19, 128.98, 128.83, 127.52, 126.06, 125.95, $121.13,116.52(\mathrm{q}, J=287.7 \mathrm{~Hz}), 112.17,110.77,108.02,100.46$, 55.90, 51.76, 47.14, 39.92, 22.23.

${ }^{19} \mathrm{~F} \mathrm{NMR}\left(376 \mathrm{MHz}, \mathrm{CDCl}_{3}\right): \delta=-68.91(2),-68.96(6),-69.02(2)$, -70.18 (1).

HRMS (ESI+): $m / z\left[\mathrm{M}+\mathrm{H}^{+}\right]$calcd for $\mathrm{C}_{23} \mathrm{H}_{22} \mathrm{~F}_{3} \mathrm{~N}_{2} \mathrm{O}_{2}: 415.1628$; found: 415.1631 .

\section{$N$-\{2-[1-Benzyl-2-(1-hydroxyallyl)-1H-indol-3-yl]ethyl\}-2,2,2-} trifluoroacetamide (12)

$\mathrm{N}$-[2-(1-Benzyl-1 $\mathrm{H}$-indol-3-yl)ethyl]-2,2,2-trifluoroacetamide (3b; 1.00 equiv, $1.00 \mathrm{~g}, 2.89 \mathrm{mmol})$ was dissolved in $\mathrm{Et}_{2} \mathrm{O}(10 \mathrm{~mL})$ and cooled to $0{ }^{\circ} \mathrm{C}$. BuLi (3.97 mL, $6.35 \mathrm{mmol}, 2.50$ equiv) was added dropwise and the reaction mixture was allowed to warm to r.t. After
$2 \mathrm{~h}$, the reaction mixture was cooled to $0{ }^{\circ} \mathrm{C}$, and acrylaldehyde $(0.212 \mathrm{~mL}, 3.18 \mathrm{mmol}, 1.50$ equiv) was added. After warming to r.t., the reaction was quenched by addition of sat. $\mathrm{Na}_{2} \mathrm{~S}_{2} \mathrm{O}_{3}(10 \mathrm{~mL})$, the mixture washed with $\mathrm{H}_{2} \mathrm{O}(3 \times 10 \mathrm{~mL})$ and extracted with $\mathrm{Et}_{2} \mathrm{O}$ $(3 \times 20 \mathrm{~mL})$. After drying over $\mathrm{MgSO}_{4}$, all volatiles were removed under reduced pressure to give the crude product, which was purified by column chromatography $\left(\mathrm{SiO}_{2}\right.$; pentane-EtOAc, $\left.7: 3\right)$ to give 12.

Yield: $0.523 \mathrm{~g}(1.299 \mathrm{mmol}, 45 \%)$; yellow solid; $R_{f}=0.90$ (pentane-EtOAc, 8:2).

${ }^{1} \mathrm{H}$ NMR $\left(400 \mathrm{MHz}, \mathrm{CDCl}_{3}\right): \delta=8.14($ br s, $1 \mathrm{H}), 7.62(\mathrm{~d}$, $J=7.6 \mathrm{~Hz}, 1 \mathrm{H}), 7.37-7.10(\mathrm{~m}, 6 \mathrm{H}), 6.97(\mathrm{~d}, J=6.6 \mathrm{~Hz}, 2 \mathrm{H}), 6.04$ (ddd, $J=17.0,10.4,5.0 \mathrm{~Hz}, 1 \mathrm{H}), 5.56(\mathrm{~d}, J=5.0 \mathrm{~Hz}, 1 \mathrm{H}), 5.44$ (d, $J=4.6 \mathrm{~Hz}, 2 \mathrm{H}), 5.33-5.11(\mathrm{~m}, 2 \mathrm{H}), 3.64(\mathrm{dd}, J=11.9,5.0 \mathrm{~Hz}$, $3 \mathrm{H}), 3.37-3.08(\mathrm{~m}, 2 \mathrm{H})$.

${ }^{13} \mathrm{C} \mathrm{NMR}\left(101 \mathrm{MHz}, \mathrm{CDCl}_{3}\right): \delta=157.52(\mathrm{q}, J=37.6 \mathrm{~Hz}), 137.81$, $137.69,136.81,135.86,128.63,127.27,127.24,125.66,122.47$, $119.71,118.37,115.84$ (q, $J=288.4 \mathrm{~Hz}), 115.52,109.84,109.69$, 67.47, 46.83, 40.60, 22.47.

${ }^{19} \mathrm{~F} \mathrm{NMR}\left(376 \mathrm{MHz}, \mathrm{CDCl}_{3}\right): \delta=-75.67$.

HRMS (ESI+): $m / z\left[\mathrm{M}+\mathrm{Na}^{+}\right]$calcd for $\mathrm{C}_{22} \mathrm{H}_{21} \mathrm{~F}_{3} \mathrm{~N}_{2} \mathrm{O}_{2} \mathrm{Na}$ : 425.1447; found: 425.1456 .

Supporting Information for this article is available online at http://www.thieme-connect.com/ejournals/toc/synthesis.

\section{Acknowledgment}

This work was financially supported by the Dutch National Research School 'combination catalysis controlled by chemical design' (NRSC-Catalysis). M.F.-M. thanks the Spanish Ministry of Science and Innovation (MICINN) for a postdoctoral fellowship.

\section{References}

(1) Cao, R. H.; Peng, W. L.; Wang, Z. H.; Xu, A. L. Curr. Med. Chem. 2007, 14, 479.

(2) Dewick, P. M. Medicinal Natural Products - A Biosynthetic Approach, 2nd ed.; Wiley: Chichester, 2001.

(3) Somei, M.; Yamada, F. Nat. Prod. Rep. 2005, 22, 73.

(4) Kochanowska-Karamyan, A. J.; Hamann, M. T. Chem. Rev. 2010, 110, 4489.

(5) Prevatt-Smith, K. M.; Prisinzano, T. E. Nat. Prod. Rep. 2010, 27, 23.

(6) Aygun, A.; Pindur, U. Curr. Med. Chem. 2003, 10, 1113.

(7) Shamon, S. D.; Perez, M. I. Cochrane Database Syst. Rev. 2009, (4), CD007655.

(8) Chen, F. E.; Huang, J. Chem. Rev. 2005, 105, 4671.

(9) Lewis, S. E. Tetrahedron 2006, 62, 8655.

(10) Bandini, M.; Eichholzer, A. Angew. Chem. Int. Ed. 2009, 48, 9608.

(11) Borschberg, H. R. Curr. Org. Chem. 2005, 9, 1465.

(12) Scholz, U.; Winterfeldt, E. Nat. Prod. Rep. 2000, 17, 349.

(13) Marques-Lopez, E.; Diez-Martinez, A.; Merino, P.; Herrera, R. P. Curr. Org. Chem. 2009, 13, 1585.

(14) Martin, S. F. Acc. Chem. Res. 2002, 35, 895.

(15) Padwa, A.; Heidelbaugh, T. M.; Kuethe, J. T.; McClure, M. S.; Wang, Q. J. Org. Chem. 2002, 67, 5928.

(16) Teichert, J. F.; Fañanás-Mastral, M.; Feringa, B. L. Angew. Chem. Int. Ed. 2011, 50, 688.

(17) Helmchen, G.; Dahnz, A.; Dübon, P.; Schelwies, M.; Weihofen, R. Chem. Commun. 2007, 675.

(18) Bartels, B.; Garcia-Yebra, C.; Helmchen, G. Eur. J. Org. Chem. 2003, 1097. 
(19) Spiess, S.; Welter, C.; Franck, G.; Taquet, J. P.; Helmchen, G. Angew. Chem. Int. Ed. 2008, 47, 7652.

(20) Kiener, C. A.; Shu, C. T.; Incarvito, C.; Hartwig, J. F. J. Am. Chem. Soc. 2003, 125, 14272.

(21) Ohmura, T.; Hartwig, J. F. J. Am. Chem. Soc. 2002, 124, 15164.

(22) Marković, D.; Hartwig, J. F. J. Am. Chem. Soc. 2007, 129, 11680.

(23) Teichert, J. F.; Feringa, B. L. Angew. Chem. Int. Ed. 2010, 49, 2486.

(24) Mingoia, Q. Gazz. Chim. Ital. 1930, 60, 509.

(25) $\mathrm{N}$-Benzyltryptamine $\mathbf{7 b}$ could also be trapped with acrolein after lithiation to give secondary allylic alcohol 12 (Scheme $6)$. See the experimental section for details.

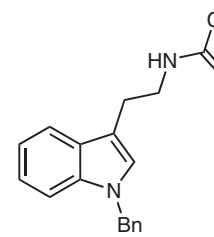

7b

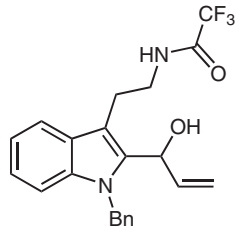

12
$45 \%$

\section{Scheme 6}

(26) Betzer, J. F.; Delaloge, F.; Muller, B.; Pancrazi, A.; Prunet, J. J. Org. Chem. 1997, 62, 7768.

(27) Shi, C.; Ojima, I. Tetrahedron 2007, 63, 8563. 